\title{
THE INFLUECE OF THE LASER ON ACOUSTIC PHONON AMPLIFICATION IN PARABOLIC POTENTIAL WELL
}

Nguyen Tien Dung ${ }^{1}$, Do Thanh Thuy ${ }^{1}$, Le Canh Trung ${ }^{1}$,

${ }^{1}$ Lab for Photonic Crystal Fiber, Vinh University, 182 Le Duan Streer, Vinh City, Viet Nam;

Email: trungch15@gmail.com

\begin{abstract}
We present a theoretical study of the kinetic equation for acoustic phonons in semiconductor quantum well with parabolic potential well under intense laser field. Using this method, we find the expression for the phonon rate coefficient for the general case, the condition of the acoustic phonon rate and the influence of the parameters of laser on this rate coefficient. We numerically calculate the rate of acoustic phonon excitation by the absorption of laser field energy at different temperature.
\end{abstract}

Keyword: phonon, quantum well, phonon rate coefficient, multiphoton absorption process.

\section{INTRODUCTION}

The development of methods for the generation and amplification of coherent acoustic phonons in low-dimensional structures [1-6]. The main results of these papers are that by absorption of laser field energy, the interaction of the laser field with electron can lead to the excitation of higher harmonics and the amplification of phonon. With the development of modern experimental technology, the fabrications of lowdimensional structures are possible [7-9]. Reality, phonon amplification by absorption of laser radiation in such confined structures would characterize the electron-phonon interaction.

The study of quantum transport theories based on the quantum kinetic equation method (QKEM) is a useful tool to investigate the multiphoton absorption process. Using the the quantum kinetic equation method, we suggested a trustful acoustic phonon excitations with a linear form [8]. The merit of using the QKEM is generalized for the two cases of the non-degenerative and degenerative electron gas can be obtained in a one-step process of expanding the phonon excitations theory.

In this paper, we start from Hamiltonian of the electron-phonon system in a Semiconductor Quantum Well (SQW) with parabolic potential under intense laser field; we derive a quantum kinetic equation for phonon in SQW in the case of multiphoton absorption process. Then, we calculate the phonon excitation rate for the two cases of the electron gas are non-degenerative and degenerative. Finally, we numerically calculate the acoustic phonon excitation rate (APER) in a specific SQW with parabolic potential to illustrate the mechanism of the phonon amplification.

\section{QUANTUM KINETIC EQUATION FOR PHONON IN A SQW}

We use a simple model for a SQW with parabolic potential $\mathrm{V}(\mathrm{z})=\frac{\mathrm{kz}^{2}}{2}$, where $\mathrm{k}$ is the force constant the oscillator. In which a two-dimensional electron gas is confined by SQW potential along the $\mathrm{z}$ 
direction and electrons are free on the $x-y$ plane. It is well known that its energy spectrum is quantized into discrete levels in the $\mathrm{z}$ direction [5].

A laser field irradiates which is normal to the $\mathrm{x}-\mathrm{y}$ plane, its polarization is along the $\mathrm{x}$ axis, and its strength is expressed as a vector potential $\vec{A}(t)$. The Hamiltonian for the system of the electrons and phonons in the case of the presence of the laser field is written as [4]:

$$
\begin{array}{r}
\hat{\mathrm{H}}(\mathrm{t})=\hat{\mathrm{H}}_{\mathrm{e}}+\hat{\mathrm{H}}_{\mathrm{ph}}+\hat{\mathrm{H}}_{\mathrm{e}-\mathrm{ph}}=\sum_{\overrightarrow{\mathrm{k}}_{\perp}, \mathrm{n}_{1}} \varepsilon_{\mathrm{n}_{1}}\left(\overrightarrow{\mathrm{k}}_{\perp}-\frac{\mathrm{e}}{\mathrm{c} \hbar} \overrightarrow{\mathrm{A}}(\mathrm{t})\right) \mathrm{a}_{\overrightarrow{\mathrm{k}}_{\perp}}^{\left(\mathrm{n}_{1}\right)^{+}} \mathrm{a}_{\overrightarrow{\mathrm{k}}_{\perp}}^{\left(\mathrm{n}_{1}\right)} \\
+\sum_{\mathrm{q}} \hbar \omega_{\overrightarrow{\mathrm{q}}} \mathrm{b}_{\overrightarrow{\mathrm{q}}}^{+} \mathrm{b}_{\overrightarrow{\mathrm{q}}}+\sum_{\overrightarrow{\mathrm{k}}_{\perp}, \overrightarrow{\mathrm{q}}, \mathrm{n}_{1}, \mathrm{n}_{2}} \mathrm{C}_{\mathrm{n}_{1}, \mathrm{n}_{2}}(\overrightarrow{\mathrm{q}}) \mathrm{a}_{\overrightarrow{\mathrm{k}}_{\perp}+\mathrm{n}_{\mathrm{q}}}^{\left(\mathrm{n}_{2}\right)^{+}} \mathrm{a}_{\overrightarrow{\mathrm{k}}_{\perp}}^{\left(\mathrm{n}_{1}\right)}\left(\mathrm{b}_{\overrightarrow{\mathrm{q}}}+\mathrm{b}_{-\overrightarrow{\mathrm{q}}}^{+}\right)
\end{array}
$$

where $a_{\overrightarrow{\mathrm{k}}_{\perp}}^{(\mathrm{n})^{+}}$and $\mathrm{a}_{\mathrm{k}_{\perp}}^{(\mathrm{n})}$ are the creation and annihilation operators of electron in the $n$ state, $\mathrm{b}_{\overrightarrow{\mathrm{q}}}^{+}$and $\mathrm{b}_{\overrightarrow{\mathrm{q}}}$ are the creation and annihilation operators of phonon, $\varepsilon_{\overrightarrow{\mathrm{q}}}=\hbar \omega_{\overrightarrow{\mathrm{q}}}$ is phonon energy for wave vector $\overrightarrow{\mathrm{q}} \cdot \varepsilon_{\mathrm{n}}\left(\overrightarrow{\mathrm{k}}_{\perp}\right)$ is the energy spectrum of the electron for the wave $\overrightarrow{\mathrm{k}}_{\perp}$, it takes the form:

$$
\varepsilon_{\mathrm{n}}\left(\overrightarrow{\mathrm{k}}_{\perp}\right)=\hbar \omega\left(\mathrm{n}+\frac{1}{2}\right)+\frac{\hbar^{2} \overrightarrow{\mathrm{k}}_{\perp}^{2}}{2 \mathrm{~m}_{\mathrm{e}}}=\varepsilon_{\mathrm{n}}+\frac{\hbar^{2} \overrightarrow{\mathrm{k}}_{\perp}^{2}}{2 \mathrm{~m}_{\mathrm{e}}} ; \text { with } \mathrm{n}=0,1,2 \ldots
$$

$e$ and $\mathrm{m}_{\mathrm{e}}$ are the charge and the effective mass of the electron, $\omega=\sqrt{\mathrm{k} / \mathrm{m}_{\mathrm{e}}} \cdot \overrightarrow{\mathrm{A}}$ is the potential vector, depend on the external field:

$$
\overrightarrow{\mathrm{A}}=\overrightarrow{\mathrm{A}}_{0} \cos \Omega \mathrm{t}, \mathrm{A}_{0}=\mathrm{cE}_{0} / \Omega .
$$

$\mathrm{C}_{\mathrm{n}_{1} \mathrm{n}_{2}}(\overrightarrow{\mathrm{q}})=\mathrm{C}_{\overrightarrow{\mathrm{q}}} \mathrm{I}_{\mathrm{n}_{1} \mathrm{n}_{2}}(\overrightarrow{\mathrm{q}})$, where $\mathrm{C}_{\overrightarrow{\mathrm{q}}}$ is the electron-phonon interaction constant and $\mathrm{I}_{\mathrm{n}_{1} \mathrm{n}_{2}}(\overrightarrow{\mathrm{q}})$ :

$$
\mathrm{I}_{\mathrm{n}_{1} \mathrm{n}_{2}}\left(\mathrm{q}_{\mathrm{z}}\right)=\int_{-\infty}^{+\infty} \mathrm{H}_{\mathrm{n}_{1}}(\mathrm{z}) \mathrm{H}_{\mathrm{n}_{2}}(\mathrm{z}) \exp \left(\mathrm{iq}_{\mathrm{z}} \mathrm{z}\right) \mathrm{dz}
$$

with $H_{n}(z)=\frac{1}{2^{n} !} \sqrt{\frac{\alpha}{\pi}}(-1)^{n} e^{\frac{\alpha z^{2}}{2}} \frac{d^{n}}{d z^{n}} e^{-\alpha z^{2}}$ is wave function.

On the basis of (1), we get the quantum kinetic equation for phonons in SQW [8].

$$
\begin{aligned}
& \frac{\partial \mathrm{N}_{\overrightarrow{\mathrm{q}}}(\mathrm{t})}{\partial \mathrm{t}}=\frac{1}{\hbar^{2}} \sum_{\overrightarrow{\mathrm{k}}, \mathrm{n}_{1}, \mathrm{n}_{2}}\left|\mathrm{C}_{\mathrm{n}_{1} \mathrm{n}_{2}}(\overrightarrow{\mathrm{q}})\right|^{2} \sum_{\ell=-\infty}^{+\infty} \mathrm{J}_{\ell}^{2}(\Lambda / \hbar \Omega) \int_{-\infty}^{\mathrm{t}} \mathrm{dt}^{\prime} \mathrm{N}_{\overrightarrow{\mathrm{q}}}\left(\mathrm{t}^{\prime}\right) \\
& \quad \times\left\{\left[\mathrm{f}_{\mathrm{n}_{2}}\left(\overrightarrow{\mathrm{k}}_{\perp}+\overrightarrow{\mathrm{q}}\right)-\mathrm{f}_{\mathrm{n}_{1}}\left(\overrightarrow{\mathrm{k}}_{\perp}\right)\right] \exp \left[\frac{\mathrm{i}}{\hbar}\left(\varepsilon_{\mathrm{n}_{2}}\left(\overrightarrow{\mathrm{k}}_{\perp}+\overrightarrow{\mathrm{q}}\right)-\varepsilon_{\mathrm{n}_{1}}\left(\overrightarrow{\mathrm{k}}_{\perp}\right)-\varepsilon_{\overrightarrow{\mathrm{q}}}-\ell \hbar \Omega\right)\left(\mathrm{t}-\mathrm{t}^{\prime}\right)\right]\right. \\
& \left.\quad+\left[\mathrm{f}_{\mathrm{n}_{1}}\left(\overrightarrow{\mathrm{k}}_{\perp}\right)-\mathrm{f}_{\mathrm{n}_{2}}\left(\overrightarrow{\mathrm{k}}_{\perp}-\overrightarrow{\mathrm{q}}\right)\right] \exp \left[-\frac{\mathrm{i}}{\hbar}\left(\varepsilon_{\mathrm{n}_{1}}\left(\overrightarrow{\mathrm{k}}_{\perp}\right)-\varepsilon_{\mathrm{n}_{2}}\left(\overrightarrow{\mathrm{k}}_{\perp}-\overrightarrow{\mathrm{q}}\right)-\varepsilon_{\overrightarrow{\mathrm{q}}}-\ell \hbar \Omega\right)\left(\mathrm{t}-\mathrm{t}^{\prime}\right)\right]\right\}
\end{aligned}
$$

where $\mathrm{N}_{\overrightarrow{\mathrm{q}}}(\mathrm{t})=\left\langle\mathrm{b}_{\overrightarrow{\mathrm{q}}}^{+} \mathrm{b}_{\overrightarrow{\mathrm{q}}}>_{t}\right.$, the symbol $<\mathrm{X}>_{\mathrm{t}}$ means the usual thermodynamic average of operator $\mathrm{X}, \mathrm{J}_{\ell}(\mathrm{z})$ is Bessel function, $\mathrm{f}_{\mathrm{n}}\left(\overrightarrow{\mathrm{k}}_{\perp}\right)$ is the distribution function of the electron, $\Lambda=\mathrm{e} \hbar \overrightarrow{\mathrm{E}}_{0} \overrightarrow{\mathrm{q}}_{\perp} /\left(\mathrm{m}_{\mathrm{e}} \Omega\right)$. 


\section{PHONON EXCITATION RATE IN A SQW}

These results allow [8] one to introduce the kinetic equation for phonon number of the q mode:

$$
\frac{\partial \mathrm{N}_{\overrightarrow{\mathrm{q}}}(\mathrm{t})}{\partial \mathrm{t}}=\gamma_{\overrightarrow{\mathrm{q}}} \mathrm{N}_{\overrightarrow{\mathrm{q}}}(\mathrm{t})
$$

where $\gamma_{\vec{q}}$ are parameters that determine the evolution of the phonon number $\mathrm{N}_{\vec{q}}(t)$ in time due to the interaction with the electrons. If $\gamma_{\vec{q}}>0$ the phonon population grows with time, whereas for $\gamma_{\vec{q}}<0$ we have damping.

Referring to Eq. (5) phonon excitation rate in (6) is:

$$
\gamma_{\overrightarrow{\mathrm{q}}}=\frac{\pi}{\hbar} \sum_{\overrightarrow{\mathrm{k}}, \mathrm{n}_{1}, \mathrm{n}_{2}}\left|\mathrm{C}_{\mathrm{n}_{1} \mathrm{n}_{2}}(\overrightarrow{\mathrm{q}})\right|^{2}\left[\mathrm{f}_{\mathrm{n}_{2}}(\overrightarrow{\mathrm{k}}+\overrightarrow{\mathrm{q}})-\mathrm{f}_{\mathrm{n}_{1}}(\overrightarrow{\mathrm{k}})\right]\left\{\delta\left(\varepsilon_{\mathrm{n}_{2}}(\overrightarrow{\mathrm{k}}+\overrightarrow{\mathrm{q}})-\varepsilon_{\mathrm{n}_{1}}(\overrightarrow{\mathrm{k}})-\varepsilon_{\overrightarrow{\mathrm{q}}}-\Lambda\right)+\delta\left(\varepsilon_{\mathrm{n}_{2}}(\overrightarrow{\mathrm{k}}+\overrightarrow{\mathrm{q}})-\varepsilon_{\mathrm{n}_{1}}(\overrightarrow{\mathrm{k}})-\varepsilon_{\overrightarrow{\mathrm{q}}}+\Lambda\right)\right\}(7)
$$

In the strong-field limit, $\Lambda>>\Omega$ and the argument of the Bessel function in Eq. (5) is larger. For large values of argument, the Bessel function is small except when the order is equal to the argument. The sum over $\ell$ in Eq. (5) may then be written approximately:

$$
\sum_{\ell=-\infty}^{\infty} \mathrm{J}_{\ell}^{2}\left(\frac{\Lambda}{\hbar \Omega}\right) \delta(\mathrm{E}-\ell \hbar \Omega)=\frac{1}{2}[\delta(\mathrm{E}+\Lambda)+\delta(\mathrm{E}-\Lambda)]
$$

Here $E=\varepsilon_{n}(\vec{k}+\vec{q})-\varepsilon_{n}(\vec{k})-\varepsilon_{\vec{q}}$. The first delta function corresponds to the absorption and the second one corresponds to the emission of $\Lambda /(\hbar \Omega)$ photons. In other words, in the strong-field limit only multiphoton processes is significant and the electron-phonon collision takes place with the emission and absorption of $\Lambda /(\hbar \Omega)$ photons. Substituting Eq. (8) into Eq. (7), the phonon excitation rate becomes $\gamma_{\vec{q}}=\gamma_{\vec{q}}^{+}+\gamma_{\vec{q}}^{-}$, where:

$$
\gamma_{\overline{\mathrm{q}}}^{( \pm)}=\frac{\pi}{\hbar_{\overrightarrow{\mathrm{k}}, \mathrm{n}_{1}, \mathrm{n}_{2}}}\left|\mathrm{C}_{\mathrm{n}_{1} \mathrm{n}_{2}}(\overrightarrow{\mathrm{q}})\right|^{2}\left[\mathrm{f}_{\mathrm{n}_{2}}(\overrightarrow{\mathrm{k}}+\overrightarrow{\mathrm{q}})-\mathrm{f}_{\mathrm{n}_{1}}(\overrightarrow{\mathrm{k}})\right] \delta\left(\varepsilon_{\mathrm{n}_{2}}(\overrightarrow{\mathrm{k}}+\overrightarrow{\mathrm{q}})-\varepsilon_{\mathrm{n}_{1}}(\overrightarrow{\mathrm{k}})-\varepsilon_{\overline{\mathrm{q}}} \pm \Lambda\right)
$$

In the following, we will calculate for the case in which the electron gas is non-degenerative. In this case, we may simplify the carrier distribution function by using the Boltzmann distribution function $\mathrm{f}_{\mathrm{n}}\left(\overrightarrow{\mathrm{k}}_{\perp}\right)=\exp \left[\beta\left(\varepsilon_{\mathrm{F}}-\varepsilon_{\mathrm{n}}\left(\overrightarrow{\mathrm{k}}_{\perp}\right)\right)\right]$. From Eq. (9), for the case in which $\overrightarrow{\mathrm{q}}=\overrightarrow{\mathrm{q}}_{\mathrm{z}}+\overrightarrow{\mathrm{q}}_{\perp}$, we obtain the expression for the rate of phonon excitations:

$$
\begin{aligned}
\gamma_{\overline{\mathrm{q}}}^{( \pm)} & =\frac{\mathrm{S}}{\hbar^{4} \mathrm{q}_{\perp}^{2}}\left(\frac{\mathrm{m}_{\mathrm{e}}^{3}}{8 \pi^{3} \beta}\right)^{1 / 2} \sum_{\mathrm{n}_{1}, \mathrm{n}_{2}}\left|\mathrm{C}_{\mathrm{n}_{1} \mathrm{n}_{2}}(\overrightarrow{\mathrm{q}})\right|^{2} \exp \left[\beta\left(\varepsilon_{\mathrm{F}}-\hbar \omega\left(\mathrm{n}_{1}+\frac{1}{2}\right)\right)\right] \\
& \times \exp \left[-\frac{\beta \mathrm{m}_{\mathrm{e}}}{2 \hbar^{2} \mathrm{q}_{\perp}^{2}}\left(\frac{\hbar^{2} \mathrm{q}_{\perp}^{2}}{2 \mathrm{~m}_{\mathrm{e}}}+\hbar \omega\left(\mathrm{n}_{2}-\mathrm{n}_{1}\right)-\hbar \omega_{\overrightarrow{\mathrm{q}}} \mp \Lambda\right)^{2}\right]\left\{\exp \left[-\beta\left(\hbar \omega_{\overline{\mathrm{q}}} \mp \Lambda\right)\right]-1\right\}
\end{aligned}
$$


Here $\beta=1 /\left(\mathrm{k}_{\mathrm{B}} \mathrm{T}\right), \mathrm{k}_{\mathrm{B}}$ is the Boltzmann constant and $\mathrm{T}$ is the temperature of the system. Analyzing Eq. (10) we can obtain the conditions for the phonon amplification. From the condition $\gamma_{\overrightarrow{\mathrm{q}}}^{( \pm)}>0$, we obtain $\exp \left[-\beta\left(\hbar \omega_{\overline{\mathrm{q}}} \mp \Lambda\right)\right]-1>0$. The condition which the laser field must satisfy is:

$$
\Lambda=\frac{\hbar \mathrm{e} \overrightarrow{\mathrm{q}}_{\perp} \overrightarrow{\mathrm{E}}_{0}}{\mathrm{~m}_{\mathrm{e}} \Omega}>\hbar \omega_{\overrightarrow{\mathrm{q}}}
$$

The condition (11) simply means that if drift velocity of electron $\overrightarrow{\mathrm{q}}_{\perp} \overrightarrow{\mathrm{E}}_{0} / \mathrm{m}_{\mathrm{e}} \Omega$ under the intense laser field, excesses the phonon phase-velocity, a deformation potential for multiphonon excitation can be generated in the SQW.

We calculate the rate of acoustic phonon excitation with $\left|\mathrm{C}_{\overrightarrow{\mathrm{q}}}\right|^{2}=\hbar \mathrm{q} \xi^{2} /\left(\rho \mathrm{v}_{\mathrm{a}} \mathrm{V}\right)$. Here, V, $\rho, \mathrm{v}_{\mathrm{a}}$, and $\xi$ are the volume, the density, the acoustic velocity, and the deformation potential constant, respectively. We have the rate of phonon excitations.

For the rate of acoustic phonon excitations:

$$
\begin{aligned}
\gamma_{\overrightarrow{\mathrm{q}}}^{(+) \mathrm{a}}= & \frac{\xi^{2}}{2 \hbar^{3} \mathrm{q}_{\perp} \mathrm{L} \rho \mathrm{v}_{\mathrm{a}}}\left(\frac{\mathrm{m}_{\mathrm{e}}^{3}}{8 \pi^{3} \beta}\right)^{1 / 2} \sum_{\mathrm{n}_{1}, \mathrm{n}_{2}}\left|\mathrm{I}_{\mathrm{n}_{1} \mathrm{n}_{2}}(\overrightarrow{\mathrm{q}})\right|^{2} \exp \left[\beta\left(\varepsilon_{\mathrm{F}}-\hbar \omega\left(\mathrm{n}_{1}+\frac{1}{2}\right)\right)\right] \\
& \times \exp \left[-\frac{\beta \mathrm{m}_{\mathrm{e}}}{2 \hbar^{2} \mathrm{q}_{\perp}^{2}}\left(\frac{\hbar^{2} \mathrm{q}_{\perp}^{2}}{2 \mathrm{~m}_{\mathrm{e}}}+\hbar \omega\left(\mathrm{n}_{2}-\mathrm{n}_{1}\right)-\hbar \omega_{\overrightarrow{\mathrm{q}}}-\Lambda\right)^{2}\right]\left\{\exp \left[-\beta\left(\hbar \omega_{\overrightarrow{\mathrm{q}}}-\Lambda\right)\right]-1\right\}
\end{aligned}
$$

In the following, we will calculate for the case in which the electron gas is degenerative. In this case, we may simplify the carrier distribution function by using the Boltzmann distribution function:

$$
\mathrm{f}_{\mathrm{n}}\left(\overrightarrow{\mathrm{k}}_{\perp}\right)=\theta\left(\varepsilon_{\mathrm{F}}-\varepsilon_{\mathrm{n}}\left(\overrightarrow{\mathrm{k}}_{\perp}\right)\right)= \begin{cases}1 & \text { if } \varepsilon_{\mathrm{F}}>\varepsilon_{\mathrm{n}}\left(\overrightarrow{\mathrm{k}}_{\perp}\right) \\ 0 & \text { if } \varepsilon_{\mathrm{F}}<\varepsilon_{\mathrm{n}}\left(\overrightarrow{\mathrm{k}}_{\perp}\right)\end{cases}
$$

For the rate of acoustic phonon excitations:

$$
\begin{aligned}
\gamma_{\overrightarrow{\mathrm{q}}}^{(+) \mathrm{a}} & =\frac{\xi^{2} \mathrm{q}^{2}}{\hbar^{3} \rho \mathrm{v}_{\mathrm{a}} \mathrm{qL}} \sqrt{\frac{\mathrm{m}_{\mathrm{e}}^{3}}{2}} \sum_{\mathrm{n}, \mathrm{n}^{\prime}}\left|\mathrm{I}_{\mathrm{n}, \mathrm{n}^{\prime}}(\overrightarrow{\mathrm{q}})\right|^{2}\left\{\left[\left(\varepsilon_{\mathrm{F}}-\varepsilon_{\mathrm{n}}-\hbar \omega_{\overrightarrow{\mathrm{q}}}+\Lambda\right)-\frac{\mathrm{m}_{\mathrm{e}}}{2 \hbar^{2} \mathrm{q}^{2}}\left(\frac{\hbar^{2} \mathrm{q}^{2}}{2 \mathrm{~m}_{\mathrm{e}}}+\hbar \omega\left(\mathrm{n}_{2}-\mathrm{n}_{1}\right)-\hbar \omega_{\overrightarrow{\mathrm{q}}}+\Lambda\right)^{2}\right]^{1 / 2}\right. \\
& \left.-\left[\left(\varepsilon_{\mathrm{F}}-\varepsilon_{\mathrm{n}}\right)-\frac{\mathrm{m}_{\mathrm{e}}}{2 \hbar^{2} \mathrm{q}^{2}}\left(\frac{\hbar^{2} \mathrm{q}^{2}}{2 \mathrm{~m}_{\mathrm{e}}}+\hbar \omega\left(\mathrm{n}_{2}-\mathrm{n}_{1}\right)-\hbar \omega_{\overrightarrow{\mathrm{q}}}+\Lambda\right)^{2}\right]^{1 / 2}\right\}
\end{aligned}
$$

The condition which the laser field must satisfy is:

$$
\varepsilon_{\mathrm{F}} \geq \varepsilon_{\mathrm{n}}+\frac{\mathrm{m}_{\mathrm{e}}}{2 \hbar^{2} \mathrm{q}^{2}}\left(\frac{\hbar^{2} \mathrm{q}^{2}}{2 \mathrm{~m}_{\mathrm{e}}}+\hbar \omega\left(\mathrm{n}_{2}-\mathrm{n}_{1}\right)-\hbar \omega_{\overrightarrow{\mathrm{q}}}+\Lambda\right)^{2}
$$




\section{NUMERICAL RESULTS AND CONCLUTIONS}

In order to clarify mechanism for the phonon amplification, we numerically calculate the rate of acoustic phonon excitation by the absorption of laser field energy. The parameters used in the calculation are as follows $\hbar=1.05 \times 10^{-34} \mathrm{Js}, \mathrm{m}_{\mathrm{e}}=0.066 \mathrm{~m}_{0}$, with $\mathrm{m}_{0}$ being the mass of free electron, $\mathrm{e}_{\mathrm{f}}=0.05 \mathrm{eV}$, $\rho=5.32 \times 10^{3} \mathrm{~kg} / \mathrm{m}^{3}, \mathrm{v}_{\mathrm{a}}=5370 \mathrm{~m} / \mathrm{s}, \xi=13.51 \mathrm{eV}, \mathrm{n}_{1}=1, \mathrm{n}_{2}=2$ for $\mathrm{GaAs} / \mathrm{Ga}_{1-x} \mathrm{As}_{x} \mathrm{Al}[10]$.

The dependence of the phonon rate increase in the case of non degenerate electron gas on the laser field amplitude with frequency value $\Omega=10^{15} \mathrm{rad} / \mathrm{s}$ at two temperatures $\mathrm{T}=100 \mathrm{~K}$ and $\mathrm{T}=150 \mathrm{~K}$ is described as Figure 1.

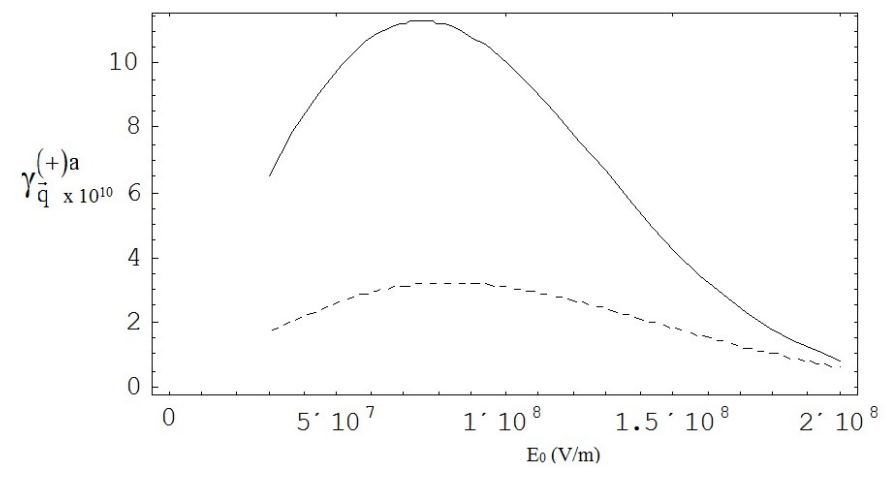

Fig 1. Dependence of phonon rate increase on laser field amplitude $E_{0}$ with frequency $\Omega=10^{15} \mathrm{rad} / \mathrm{s}$, wave number $\mathrm{q}=2 \times 10^{8} \mathrm{~m}^{-1}$, here $\mathrm{T}=100 \mathrm{~K}$ solid curve and $\mathrm{T}=150 \mathrm{~K}$ broken curve.

The dependence of the rate of phonon increase in the case of non degenerate electron gas on $\Omega$ laser frequency with the laser field amplitude $\mathrm{E}_{0}=2.3 \times 10^{7} \mathrm{~V} / \mathrm{m}$ at two temperatures $\mathrm{T}=100 \mathrm{~K}$ and $\mathrm{T}=150 \mathrm{~K}$ is described in Figure 2.

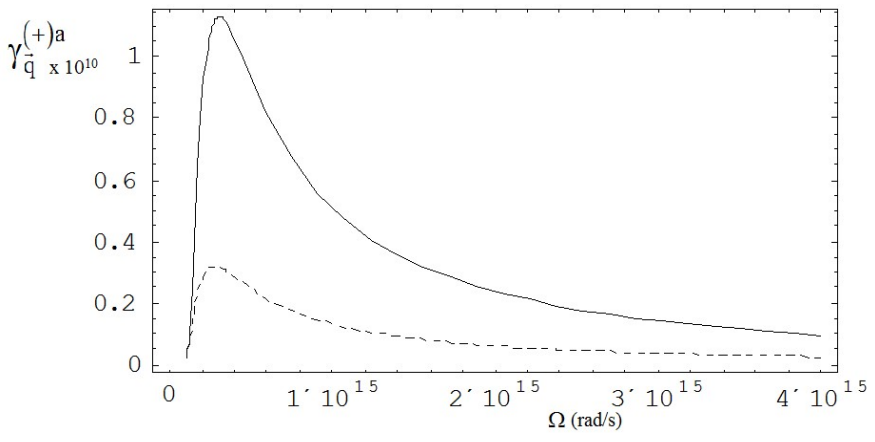

Fig 2. Dependence of phonon rate increase on laser field frequency $\Omega$ with laser field amplitude $E_{0}$ $=2.3 \times 10^{7} \mathrm{~V} / \mathrm{m}$, wave number $\mathrm{q}=2 \times 10^{8} \mathrm{~m}^{-1}$, here $\mathrm{T}=100 \mathrm{~K}$ solid curve and $\mathrm{T}=150 \mathrm{~K}$ broken curve.

Figures 1 and 2 show the existence of field strength and frequency values for maximum phonon growth rates. Comparing the graphs in Figures 1 and 2 show that when the frequency increases, the rate of increase of phonon increases faster to the maximum value then decreases. The higher the temperature, the 
lower the maximum value, meaning that the number of negative phonons produced decreases. In our opinion, the reason for this is that the higher the temperature, the greater the phonon energy, while the field energy is constant, so the rate of negative phonon production decreases. The sensitivity of the rate of phonon production to temperature is a characteristic of negative phonons.

The dependence of the rate of phonon increase in the case of degenerate electron gas on the laser frequency A and the B laser field amplitude as shown in Figure 3.

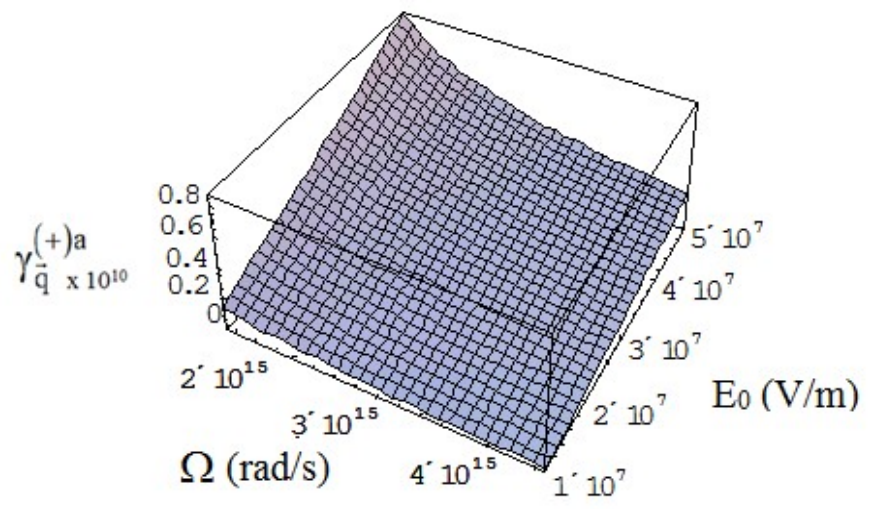

Fig 3. Dependence of phonon rate increase on laser field frequency $\Omega$ and laser field amplitude, at wave number $\mathrm{q}=2 \times 10^{8} \mathrm{~m}^{-1}$.

From Figure 3, we see that as the frequency and amplitude of the laser field increase, in the case of degenerate electron gas, the rate of increase of phonon increases

In conclusion, we have analytically investigated the possibility of phonon amplification by absorption of laser field energy in a SQW with parabolic potential well in the case of multiphoton absorption process with non-degenerative and degenerative electron system. Starting from bulk phonon assumption and Hamiltonian of the electron-phonon system in laser field we have derived a quantum kinetic equation for phonon in SQW. However, an analytical solution to the equation can only be obtained within some limitations. Using these limitations for simplicity, we have obtained expressions of the rate of acoustic phonon excitation in the case of multiphoton absorption process. Finally, the expressions are numerically calculated and plotted for a SQW to show the mechanism of the phonon amplification. Similarly to the mechanism pointed out by several authors for different models, phonon amplification in a SQW can occur under the conditions that the amplitude of the external laser field is higher than some threshold amplitude as the condition of the Cerenkov.

\section{REFERENCES}


[1]. O. A. C. Nunes and A. L. A. Fonseca, Journal of Applied Physics, 112, 043707, (2012).

[2] C. X. Zhao, W. Xu and F. M. Peeters, Applied physics letters, 102, 222101, (2013).

[3]. O. A. C. Nunes, Journal of Applied Physics, 115, 233715, (2014).

[4]. K. A. Dompreh, N. G. Mensah, S. Y. Mensah, and S. K. Fosuhene, Low Temperature Physics, 42, 466, (2016).

[5]. Nguyen Tien Dung, CASEAN-6 Proceedings, 234-239, (2019)

[6] Nguyen Tien Dung, Le Canh Trung, Pham Thi Hoai Duong, Tran Cong Phong, Photonics Letters of Poland, 13 (1), 7-9 (2021)

[7]. Derkacs, D. Chen, W. V. Matheu, P. M. Lim, S. H.Yu, P. K. L. Yu, E. T, Applied Physics Letters, 93 (9): 091107, (2008).

[8]. Ramey, S. M.; Khoie, R, IEEE Transactions on Electron Devices, 50 (5): 1179-1188, (2003).

[9]. Pallab Bhattacharya, Roberto Fornari and Hiroshi Kamimura, Comprehensive Semiconductor Science and Technology, Pages 256-342, (2011).

[10]. S.M. Komirenko, K.W. Kim, A.A. Dimidenko, V.A. Kochelap, and M.A. Stroscio, Physcal Review B, B62, 7459,(2000). 